\title{
Oral Mucositis, Pain and Xerostomia in Patients with Head and Neck Cancer who Received Chemoradiotherapy with or without Cetuximab
}

\author{
Ourania Nicolatou-Galitis ${ }^{*}$, Triantafyllia Sarri ${ }^{1}$, Konstantinos Dardoufas ${ }^{2}$, Vassilis Kouloulias ${ }^{3}$, \\ Xenophon Vakalis ${ }^{4}$, Argyro Polychronopoulou ${ }^{5}$, Dimitrios Demenagas ${ }^{6}$ and \\ Anastasia Sotiropoulou-Lontou ${ }^{6}$
}

${ }^{I}$ Clinic of Hospital Dentistry, Dental Oncology Unit, School of Dentistry, University of Athens, Greece

${ }^{2}$ Department of Radiotherapy, "Hygeia” Hospital, Athens, Greece

${ }^{3}$ Department of Radiotherapy, “Attikon” Hospital, School of Medicine, University of Athens, Greece

${ }^{4}$ Department of Radiotherapy, "401 General Militairy Hospital”, Athens, Greece

${ }^{5}$ Department of Preventive and Community Dentistry, School of Dentistry, University of Athens, Greece

${ }^{6} 2^{\text {nd }}$ Radiotherapy Department, "O Aghios Savvas" Hospital, Athens, Greece

\begin{abstract}
Goal of work: To compare the severity of oral mucositis, pain and xerostomia in head and neck cancer patients, who received radiotherapy with cisplatin and cetuximab to that of patients who received radiotherapy with cisplatin alone.

Patients: Forty-nine head and neck cancer patients entered the study. Twenty-five patients (Group A) received radiotherapy and cisplatin. Twenty-four patients (Group B) received radiotherapy, cisplatin, and cetuximab.

Methods: Oral mucositis was recorded weekly, according to EORTC/RTOG criteria. Pain and xerostomia were assessed using a $10 \mathrm{~cm}$ visual analogue scale. Antifungal and antiviral treatment and prophylaxis were administered during RT to both groups.

Results: During chemoradiotherapy, severe mucositis, pain and xerostomia were observed in $60 \%, 64 \%$ and $52 \%$ respectively in Group A, while the same symptoms were observed in $79 \%, 58 \%$ and $29 \%$ respectively in Group B. The differences between the two groups were not statistically significant. At the end of chemoradiotherapy, severe mucositis, pain and xerostomia were recorded in 24\%, 32\% and 32\% in Group A and 37\%, 21\% and 17\% respectively in Group B. The differences between the two groups were, again, statistically not significant. Neither significant differences were found between the two groups with respect to the use of antifungal and antiviral treatment, radiotherapy interruptions and weight loss. In both groups, oral mucositis, pain and xerostomia were significantly reduced at the end of radiotherapy as compared to those during RT, following the anti-infectious treatment and prophylaxis.
\end{abstract}

Conclusion: Cetuximab, added to cisplatin/radiotherapy, did not increase the severity of oral mucositis, pain and xerostomia, in head and neck cancer patients, with limitations of the study design and its limited number of patients.

Keywords: Cetuximab, head and neck cancer, mucositis, pain, radiotherapy, xerostomia.

\section{INTRODUCTION}

Chemotherapy (Chemo) has become an integral part of potentially curative therapy for head and neck cancer. Chemoradiotherapy (ChemoRT) has been shown to be superior over radiotherapy (RT) alone, in terms of locoregional control and overall survival. Platinum based regimens in combination with 5-fluorouracil have been the most effective chemotherapy regimen so far. Given the toxicity of concurrent ChemoRT, careful selection of patients is critical [13].

*Address correspondence to this author at the School of Dentistry, University of Athens, Bouboulinas 41, N. Psyhico, 15451 Athens, Greece; Tel: +30 210 6748715; Fax: +30 2106775567 ;

E-mail: nicolatou.galitis@lycos.com
Targeted therapies, which include monoclonal antibodies and small molecule inhibitors, that target specific growth factors and growth factor receptors, have significantly changed the treatment of cancer over the past 10 years. One particular growth factor receptor and signal transduction system that has been shown to play an important role in the pathogenesis of head and neck cancer is the epidermal growth factor receptor (EGFR) and its ligand. Ligand binding to EGFR results in activation of tyrosine kinase pathways that significantly affect tumorigenesis by stimulating cell proliferation, inhibiting apoptosis and favoring angiogenesis to facilitate metastasis.

EGFR is overexpressed (80-100\%) and/or abnormally activated in head and neck cancers, correlating with poor clinical outcome in these patients [4-6]. Inhibition of the 
EGFR-induced signal transduction pathways by monoclonal antibodies has been shown to inhibit the growth of EGFRexpressing human cancer cells.

Cetuximab (C-225, Erbitux $\left.{ }^{\circledR}\right)$, is an IgG1 subclass mouse-human chimeric monoclonal antibody, which binds with high affinity to the extracellular ligand binding domain of the receptor, leading to inhibition of cancer cell proliferation, invasion and migration.

Cetuximab competes with natural legands of EGFR for binding to the receptor and thus, prevents activation of the receptor. In addition, cetuximab might trigger the internalization and degradation of the receptor. An antineoplastic effect mediated by immune mechanisms has also been postulated, specifically by the induction of antibody-dependent cellmediated cytotoxicity [7].

On the other hand, EGFR is also expressed in normal epithelial cells (of skin and mucosa), thus, EGFR inhibition can lead to significant dermatologic and gastrointestinal toxicities.

Several studies and clinical trials have been conducted to investigate the effect and safety of cetuximab used for the treatment of locoregionally advanced and/or recurrent or metastatic squamous cell carcinoma of the head and neck or nasopharyngeal carcinoma, in terms of locoregional control, free-disease survival, and overall survival [8-17].

Cetuximab has been used alone and in combination with platinum-based chemotherapy [9-11, 13] or in combination with platinum-based chemotherapy and 5-fluorouracil (5FU) $[12,14,17]$. Cetuximab has also been used in combination with radiotherapy $[8,15,16]$.

Cetuximab has been found to be well tolerated and active. A superior overall survival, progression-free survival and locoregional control, attributed to cetuximab, have been reported [14-17].

When cetuximab was added to platinum-based chemotherapy and 5-FU, there was no significant difference in the overall incidence of grade 3 or 4 adverse events between the groups [14]. The addition of cetuximab had no impact on the Quality of Life, as it was assessed with the QLQ-C30 and QLQ-H\&N35 questionnaires. Furthermore, patients in the cetuximab arm displayed significant improvements in pain, swallowing problems and scores for speech and social eating problems [17].

Skin reactions, particularly an acne-like rash, as a result of the normal expression of EGFR in the skin, are the most common cetuximab-related adverse events, in cetuximad and chemotherapy combinations, reported in more than half of the patients, mainly grade 1 and 2 [9-14, 17].

The combination of cetuximab and radiotherapy in patients with locoregionally advanced head and neck carcinoma, did not increase the common toxic effects associated with RT, including oral mucositis, xerostomia, dysphagia, oral pain/odynophagia, weight loss and performance status deterioration $[8,15,16]$.

The addition of cetuximab did not adversely affect the quality of life. This was particularly notable for global health status/QoL, social functioning, social eating and social contact [16]. Acneiform rash was found to cause a dose reduction in less than $5 \%$ of the patients.

In contrast to the previously documented efficacy and safety of cetuximab, Pryor et al. [18] reported that the addition of cetuximab to radiotherapy, administered to 13 patients, with locally advanced head and neck carcinoma and major co-morbidities, including dysphagia and significant weight loss prior to the commencement of therapy, resulted in a high rate of toxicity, associated with low treatment compliance and delays in completing RT. Grade 3 oral mucositis developed in 10 patients $(77 \%)$ frequently in areas that had received less than $10-15 \mathrm{~Gy}$, most commonly the mucocutaneous junction of the lips.

The mucocutaneous junction of the lips is, however, the site of predilection of herpes simplex virus-1 (HSV-1) reactivation and infection (Herpes Labialis). Herpes simplex virus-1 reactivation and infection is frequent in cancer patients [19] and can, often, complicate the ulcers of oral mucositis, increasing the severity of mucositis $[20,21]$. Accordingly, HSV-1 infection could be related to the severe mucositis on the mucocutaneous junction of the lips in those 10 patients reported by Pryor et al. [18].

Significant adverse effects, including one myocardial infarction, one bacteremia, one atrial fibrillation, and two deaths (one of pneumonia) were also reported when cetuximab was administered concurrently with radiotherapy and platinum-based chemotherapy in 22 patients, with predominantly stage IV squamous cell carcinomas of the head and neck, although preliminary efficacy was encouraging [22]. The study was closed because of significant adverse events. The potential microbial systemic dissemination from the oral cavity in patients with advanced cancers and co-morbidities was not considered. As it is known, the oral mucosal barrier damage and the development of oral ulcerative mucositis may allow for the development of local and / or systemic fungal or viral or bacterial infections.

Severe oral ulcerative mucositis, grade 3 or 4 , is a major limitation to continuous, uninterrupted RT and concurrent chemotherapy in the management of head and neck cancer. Nearly all patients (90-97\%) with head and neck cancers receiving RT or chemoRT will experience some degree of mucositis [23-25]. Over $50 \%$ of these patients may experience severe, grade 3-4 mucositis, associated severe pain, dysphagia and severe weight loss, which compromises patient's quality of life. Higher grades of ulcerative mucositis are also related to higher frequency of radiation treatment breaks with adverse effect on tumor control $[26$, 27].

In addition, mucositis has a major economic impact due to costs associated with pain management, liquid diet supplements, gastrostomy tube placements or total parenteral nutrition, management of secondary infections and hospitalizations $[28,29]$.

Local infections, candidiasis and herpes simplex may complicate the clinical course of oral mucositis, while oral flora colonizing the mucosa may lead to secondary potentially life threatening systemic infection [30-32], especially in compromised patients, with advanced or metastatic cancer. 
The most common and well studied infection of the oral mucosa during head and neck cancer RT is oral candidiasis, reported with an incidence of 27\%-53\% [20, 33-36].

Oral candidiasis develops superimposed on mucostis, in a Candida carrier, potentially adding to the severity of mucositis. Candida carriage prevalence, in head and neck RT, varies between $50 \%$ to $70 \%$ [36-38]. Systemic administration of fluconazole prophylaxis has prevented candidiasis, and has significantly reduced the Candida carriage and the severity of oral mucositis and RT interruptions [36, 39, 40].

Herpes simplex virus-1 (HSV-1) reactivation, both before and after cancer therapy, has been reported to be frequent. Patients, who received cancer therapy and were found HSV positive with direct immunofluorescence, seemed to have more severe oral mucositis than HSV negative patients, while a positive IgM result was more frequent in the mucositis group of patients [19].

Herpes simplex virus-1 infection in radiation-induced oral mucositis, complicating the severity of mucositis has been shown in our head and neck cancer patient population $[20,21]$ with an incidence of $29 \%$. HSV-1 infection has been observed to aggravate mucositis.

Based on the presented data of the high incidence of candidiasis, and Candida carriage, the risk of infection recurrences, as well as the high risk of HSV-1 reactivation and infection and the infections' potential role in the severity of oral mucositis, combined with the difficulty in the differential diagnosis of mucositis from infections [21, 36], systemic antifungal and antiviral treatment and prophylaxis has been introduced to our head and neck cancer patient population as a routine clinical practice, since 2005 .

The purpose of this report was the assessment of oral toxicity in head and neck cancer patients who received cetuximab, added to radiotherapy and platinum-based chemotherapy. The oral toxicity was prospectively evaluated in 24 head and neck patients and was compared to that of 25 patients, who received RT with platinum-based chemotherapy alone, in the years 2007 and 2008. The patients, in both arms, received antifungal and antiviral treatment and prophylaxis.

\section{PATIENTS AND METHODS}

\section{Patients and Eligibility Criteria}

Forty-nine consecutive patients with malignant head and neck tumor, eligible to receive chemoradiotherapy were included in the study. The patients were reffered to the Dental Oncology Unit for standard oral oncology supportive care before and during the course of chemoradiotherapy from four Athens Cancer Centers between January 2007 to September 2008.

General blood tests, liver and renal functions were within normal limits. Karnofsky perfomance status ranged between $80-100 \%$.

Twenty-five patients (Group A) received RT and concomitant chemotherapy, which consisted of cisplatin $75 \mathrm{mg} / \mathrm{m}^{2}$ every three weeks.
Twenty-four patients (Group B) received RT and concomitant chemotherapy, which consisted of cisplatin $75 \mathrm{mg} / \mathrm{m}^{2}$ every three weeks and cetuximab at an initial dose of $400 \mathrm{mg} / \mathrm{m}^{2}$, followed by $250 \mathrm{mg} / \mathrm{m}^{2}$ every two weeks.

The decision whether the patient would receive RT and cisplatin or RT, cisplatin and cetuximab relied upon each patient's radiation oncologist.

Patient data are summarized in Table $\mathbf{1 .}$

The median age was 63 years (range $42-83$ years) for Group A (RT plus cisplatin) and 57 years (24-78 years) for Group B (RT plus ciplatin and cetuximab). The majorities of the patients were male in both groups $(68 \%$ and $71 \%$, respectively) and had squamous cell carcinoma according to histological diagnosis (56\% and $46 \%$, respectively). In addition, the majority of our patients were in $\mathrm{T} 1$ and $\mathrm{T} 2$ of their cancer ( $76 \%$ of Group A and $62.5 \%$ of Group B).

The two groups did not differ significantly in respect to most of the baseline characteristics of the patients. Significantly more patients from Group B were, however, free of nodal disease.

All patients provided written informed consent.

\section{ChemoRadiotherapy}

Patients were irradiated with a 6-MV linear accelerator. Twenty-five patients received radical and 24 patients received postoperative radiotherapy. The primary tumor and draining lymphatics were treated with parallel-opposed fields. Supraclavicular and low-neck nodes were treated with an anterior field.

The daily and the total radiation dose are shown in Table 1.

In Group A the majority of the patients (68\%) received a daily dose of $1.8 \mathrm{~Gy}$. The mean total dose was $65.6 \mathrm{~Gy}$ (range 59,4 to $72 \mathrm{~Gy}$ ).

In Group B the majority of the patients (83\%) received a daily dose of $2.0 \mathrm{~Gy}$. The mean total dose was $64.6 \mathrm{~Gy}$ (range 46 to $70 \mathrm{~Gy}$ ). Significantly more patients from Group $\mathrm{B}$ were irradiated with a daily dose of $2.0 \mathrm{~Gy}$.

The lateral field doses were reduced after 40-43 Gy to avoid overdose to the spinal cord. The regional nodes were irradiated to a total dose of 45-61 Gy, depending on the nodal stage.

Group A (RT and cisplatin) received concomitant cisplatin at a dose of $75 \mathrm{mg} / \mathrm{m}^{2}$ every three weeks.

Group B (RT plus cisplatin and cetuximab) received concomitant cisplatin at a dose of $75 \mathrm{mg} / \mathrm{m}^{2}$ every three weeks and cetuximab at an initial dose of $400 \mathrm{mg} / \mathrm{m}^{2}$, followed by $250 \mathrm{mg} / \mathrm{m}^{2}$ every two weeks.

\section{Oral Clinical Evaluation}

Data on oral toxicity were prospectively collected, outside the context of a clinical trial. Patients were examined weekly.

Oral mucosal evaluation was performed by the oral medicine specialist and mucosal evaluation included: 
Table 1. Patient Demographics, Tumor Type, Dose of RT $(n=49)$

\begin{tabular}{|c|c|c|c|c|c|}
\hline \multirow{2}{*}{ PARAMETER } & \multicolumn{2}{|c|}{ Group A (n=25) } & \multicolumn{2}{|c|}{ Group B (n=24) } & \multirow{2}{*}{ p-value } \\
\hline & $\mathbf{N}$ & $\%$ & $\mathbf{N}$ & $\%$ & \\
\hline \multicolumn{6}{|l|}{ Gender } \\
\hline Male & 17 & 68 & 17 & 70.8 & $\mathrm{NS}^{\#}$ \\
\hline Female & 8 & 32 & 7 & 29.2 & \\
\hline \multicolumn{6}{|l|}{ Age } \\
\hline Mean & 62.8 & & 56.6 & & $\mathrm{NS}^{+}$ \\
\hline Range & $42-83$ & & $24-78$ & & \\
\hline \multicolumn{6}{|l|}{ Tumor histological diagnosis } \\
\hline Scca & 14 & 56 & 11 & 45.8 & $\mathrm{NS}^{\#}$ \\
\hline Npca & 6 & 24 & 9 & 37.5 & \\
\hline Other & 5 & 20 & 4 & 16.7 & \\
\hline \multicolumn{6}{|l|}{ Tumor stage $(N=24)$} \\
\hline $\mathrm{T} 1$ & 1 & 4.2 & 4 & 16.7 & NS* \\
\hline $\mathrm{T} 2$ & 18 & 75 & 11 & 45.8 & \\
\hline $\mathrm{T} 3$ & 1 & 4.2 & 4 & 16.7 & \\
\hline $\mathrm{T} 4$ & 2 & 8.3 & 4 & 16.7 & \\
\hline $\mathrm{Tx}$ & 2 & 8.3 & 1 & 4.2 & \\
\hline \multicolumn{6}{|l|}{ Node stage $(N=24)$} \\
\hline No & 2 & 8.3 & 8 & 33.3 & $\mathrm{p}=0.03 *$ \\
\hline $\mathrm{N} 1$ & 12 & 50 & 7 & 29.2 & \\
\hline N2 & 10 & 41.7 & 6 & 25 & \\
\hline N3 & 0 & 0 & 3 & 12.5 & \\
\hline $\mathrm{Nx}$ & 0 & 0 & 0 & 0 & \\
\hline \multicolumn{6}{|l|}{ Type of $R T$} \\
\hline Radical & 14 & 56 & 11 & 45.8 & $\mathrm{NS}^{\#}$ \\
\hline Postoperative & 11 & 44 & 13 & 54.2 & \\
\hline \multicolumn{6}{|l|}{ Daily dose } \\
\hline $1.8 \mathrm{~Gy}$ & 17 & 68 & 3 & 12.5 & $\mathrm{p}<0.003^{*}$ \\
\hline $2.0 \mathrm{~Gy}$ & 8 & 32 & 20 & 83.3 & \\
\hline $2.4 \mathrm{~Gy}$ & 0 & 0 & 1 & 4.2 & \\
\hline \multicolumn{6}{|l|}{ Total dose } \\
\hline Mean & 65.6 & & 64.6 & & $\mathrm{NS}^{+}$ \\
\hline Range & $59.4-72$ & & $46-70$ & & \\
\hline
\end{tabular}

\#: p-value based on Pearson chi square test

$+:$ p-value based on t-test

*: p-value based on Fisher's exact test

NS: non-significant

1. The scoring of oral mucositis, according to EORTC/ RTOG criteria as follows: Grade 1 (diffuse erythema, patient can eat solid food), Grade 2 (erythema and small foci or ulcers, patient can take soft diet), Grade 3 (painful ulcers extending on more than half of the oral mucosa, patient can take liquids only), Grade 4 (painful ulcers covering almost all mucosal surfaces, alimentation is not possible).

2. The presumptive diagnosis of oral pseudomembranous candidiasis, which was made, as described before [20, 35 , 36], when easily removable, mostly painless, whitish pseudomembranes were observed. 
3. The presumptive diagnosis of HSV-1 infection, which was evaluated according to the clinical criteria previously reported $[20,21]$. Those criteria included: (i) abrupt appearance of severe, extensive ulcers and/or (ii) early initiation of ulceration, within the first 2 weeks of RT, ulcers on the dorsum of the tongue, or on the hard palate, or on the vermillion border.

According to the policy of our Clinic, antifungal and antiviral treatment and prophylaxis, based on clinical criteria, as described, were administered. Antifungal and/or antiviral treatment was administered according to the drug's instructions

Antifungal treatment consisted of itraconazole $200 \mathrm{mg} /$ day for one week or posaconazole $200 \mathrm{mg}$ the first day and $100 \mathrm{mg} /$ day for another 13 days.

Antiviral treatment consisted of acyclovir $2 \mathrm{gr}$ per day for one week.

Antifungal and antiviral medication continued as prophylaxis, reduced to the half dose, until the end of RT.

Standard oral mucosal and dental care, including blant mouthrinses and topical fluorides, were introduced to all patients.

Pain and xerostomia were self-assessed using a $10 \mathrm{~cm}$ visual analogue scale by the patients. A score from 0 to 4 was evaluated as mild pain or xerostomia, a score from 5 to 7 was evaluated as moderate pain or xerostomia and a score from 8 to 10 was evaluated as severe pain and xerostomia.

\section{Statistical Analysis}

hi-squared test was employed to compare the two groups of interest, with respect to baseline characteristics, incidence of severe mucositis, pain and xerostomia during and after the $\mathrm{RT}$, the use of antifungals and antivirals, the incidence of interruptions overall and the incidence of interruptions due to severe mucositis, and the weight loss. Total dose and age were assessed by t-test while daily dose, tumor and node stage were assessed by Fisher's exact test.

The prevalence of severe mucositis, severe pain and severe xerostomia at the end of RT was assessed by McNemar's chi-squared test.

All statistical tests were two-sided, and the level of statistical significance was set at 5\%.

\section{RESULTS}

\section{ChemoRadiotherapy}

\section{Group A (RT and cisplatin)}

Twenty-one of 25 patients (84\%) completed chemoradiotherapy within the preplanned time. Four patients $(16 \%)$ interrupted RT for one week; three of them had a treatment gap due to severe mucositis (Table 2).

\section{Group B (RT, cisplatin and cetuximab)}

Nineteen of 24 patients $(79,2 \%)$ completed chemoradiotherapy within the preplanned time. Five patients $(20.8 \%)$ interrupted their therapy for one week; four of them had a treatment gap because of severe mucositis (Table 2 ).
The difference of RT interruption due to severe mucositis between the two groups was not found significant.

\section{Skin Reactions in Group B}

Fifteen of 24 patients developed severe skin toxicity, including dry desquamation, early moist desquamation, blister formation, skin pealing and bleeding ulcers. Patients were encouraged to maintain good standards of hygiene following the consensus guidelines [41]. They were treated with topical application of fucidic acid and betamethazone (Fucicort Cream) and/or betamethazone valerate (Betnovate cream), twice a day. Skin toxicity was well tolerated and no one patient discontinued therapy because of severe skin reactions.

\section{Oral Toxicity During the Course of RT (Table 2)}

\section{Oral Mucositis}

\section{Group A:}

Twenty-two of 25 patients (88\%) developed ulcerative mucositis (grade 2, 3 or 4).

Mild to moderate mucositis was evaluated in 10 patients $(40 \%)$, while severe mucositis grade 3 or 4 was scored in 15 patients $(60 \%)$, as it is shown in Table 2 .

\section{Group B:}

Twenty-three of 24 patients (96\%) developed grade 2, 3 or 4 mucositis.

Mild to moderate mucositis was evaluated in 5 patients $(20.8 \%)$, while severe mucositis grade 3 or 4 was scored in 19 patients $(79.2 \%)$, as it is shown in Table 2 .

The incidence of severe mucositis during RT between the two groups did not differ statistically.

\section{Oral Pain}

Mild and moderate pain was reported by 9 of 25 patients $(36 \%)$ in Group A and by 10 of 24 patients (41.7\%) in Group B.

Severe pain was reported by 16 of 25 patients $(64 \%)$ in Group A and by 14 of 24 patients (58.3\%) in Group B.

The incidence of severe pain between the two groups during RT was not statistically significant.

\section{Xerostomia}

Mild and moderate xerostomia was reported by 12 out of 25 patients (48\%) in Group A and by 17 out of 24 patients $(70 \%)$ in Group B.

Severe xerostomia was reported by 13 of 25 patients $(52 \%)$ in Group A and by 7 of 24 patients (30\%) in Group B. The incidence of severe xerostomia between the two groups during RT was not statistically significant.

\section{Antifungals}

Systemic antifungals were used in 20 patients $(80 \%)$ of Group A and in another 20 patients (83\%) of Group B. 
Table 2. Mucositis, Pain and Xerostomia During Chemo-RT. $(n=49)$

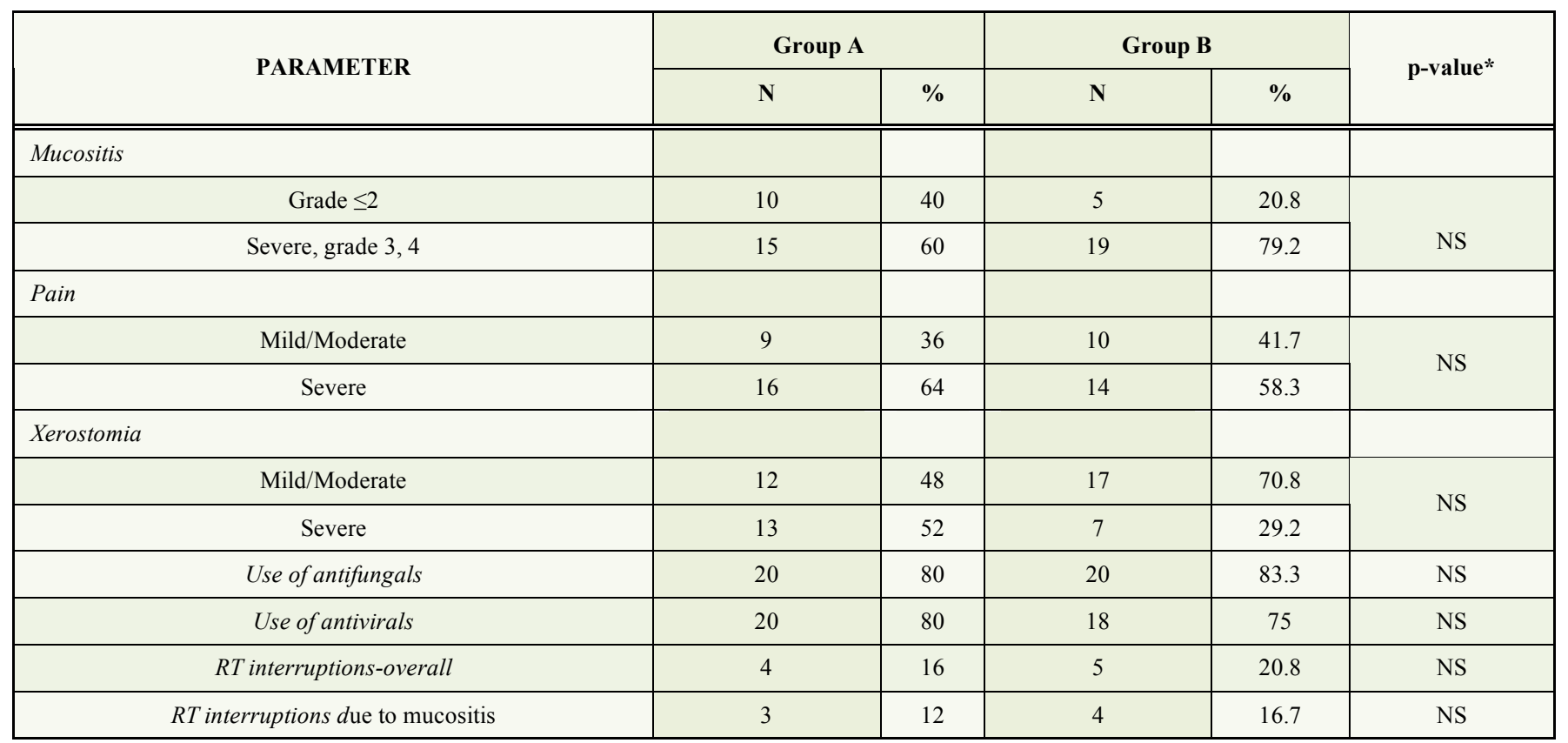

*based on Chi-square test

Oral toxicity following the completion of RT (Table 3 and Table 4)

\section{Antivirals}

Systemic antivirals were administered to 20 patients (80\%) of Group A and to 18 patients (75\%) of Group B.

The differences in antifungal and antiviral use between the two Groups were not significant.

\section{Oral Toxicity Following the Completion of RT (Tables 3 and Table 4)}

\section{Oral Mucositis}

\section{$\underline{\operatorname{Group} A}$}

Overall ulcerative mucositis grade 2, 3 and 4 was evaluated in 19 patients $(76 \%)$.

Mild to moderate mucositis was evaluated in 19 patients (76\%), while the incidence of severe mucositis grade 3 or 4 was significantly reduced to $24 \%$, observed in 6 patients, as it is shown in Table 3 and $4(p=0.003)$.

\section{Group B}

Overall ulcerative mucositis grade 2, 3 and 4 was evaluated in 19 patients $(79.2 \%)$.

Mild to moderate mucositis was evaluated in 15 patients $(62.5 \%)$, while the incidence of severe mucositis grade 3 or 4 was significantly reduced to $37.5 \%$, observed in 9 patients, as it is shown in Tables 3 and $\mathbf{4}(\mathrm{p}=0.002)$.

The differences between the two groups in terms of the incidence of severe mucositis at the end of RT were not statistically significant.

The reduction of the severity of mucositis, attributed to the antifungal and antiviral prophylaxis, at the end of RT, was significant in both groups $\mathrm{A}$ and $\mathrm{B}$.

\section{Oral Pain}

Mild and moderate pain was reported by 17 patients $(68 \%)$ in Group A and by 19 patients $(79.2 \%)$ in Group B.

Table 3. Mucositis, Pain and Xerostomia after Chemo-RT. $(n=49)$

\begin{tabular}{|c|c|c|c|c|c|}
\hline \multirow{2}{*}{ PARAMETER } & Group A & & Group B & & \multirow{2}{*}{ p-value* } \\
\hline & $\mathbf{N}$ & $\%$ & $\mathbf{N}$ & $\%$ & \\
\hline \multicolumn{6}{|l|}{ Mucositis } \\
\hline Grade $\leq 2$ & 19 & 76 & 15 & 62.5 & \multirow[b]{2}{*}{ NS } \\
\hline Severe, grade 3,4 & 6 & 24 & 9 & 37.5 & \\
\hline \multicolumn{6}{|l|}{ Pain } \\
\hline Mild/Moderate & 17 & 68 & 19 & 79.2 & \multirow{2}{*}{ NS } \\
\hline Severe & 8 & 32 & 5 & 20.8 & \\
\hline \multicolumn{6}{|l|}{ Xerostomia } \\
\hline Mild/Moderate & 17 & 68 & 20 & 83.3 & \multirow{2}{*}{ NS } \\
\hline Severe & 8 & 32 & 4 & 16.7 & \\
\hline Loss of weight & $\mathrm{N}=17 * *$ & & $\mathrm{~N}=18$ & & \\
\hline Median & $9,8 \mathrm{~kg}$ & & $8,6 \mathrm{~kg}$ & & \\
\hline Range & $2-29$ & & $2-15$ & & \\
\hline
\end{tabular}

*based on Chi-square test

** 1 patient did not lose weight

The prevalence of severe pain was significantly reduced to $32 \%$ in Group A and to $20.8 \%$ in Group B, $\mathrm{p}=0.003$.

The differences between the two groups in terms of the prevalence of severe pain at the end of RT were not statistically significant. 


\section{Xerostomia}

Mild and moderate xerostomia was reported by 17 patients (68\%) in Group A and by 20 patients $(83.3 \%)$ in Group B.

In Group A severe xerostomia was significantly reduced to $32 \%, \mathrm{p}=0.03$. In Group B xerostomia was also reduced, though not significantly, to $16.7 \%$.

The differences between the two groups in terms of the prevalence of severe xerostomia at the end of RT were not statistically significant.

Table 4. Mucositis, Pain and Xerostomia During and after Chemo-RT, in Group A and in Group B

\begin{tabular}{|c|c|c|c|c|c|}
\hline \multirow[b]{2}{*}{ Group A $(n=25)$} & \multicolumn{2}{|c|}{ During RT } & \multicolumn{2}{|c|}{ After RT } & \multirow[b]{2}{*}{ p-value* } \\
\hline & $\mathbf{N}$ & $\%$ & $\mathbf{N}$ & $\%$ & \\
\hline \multicolumn{6}{|l|}{ Mucositis } \\
\hline Grade $\leq 2$ & 10 & 40 & 19 & 76 & \multirow{2}{*}{0.003} \\
\hline Grade 3,4 & 15 & 60 & 6 & 24 & \\
\hline \multicolumn{6}{|l|}{ Pain } \\
\hline Mild/moderate & 9 & 36 & 17 & 68 & \multirow{2}{*}{0.005} \\
\hline Severe & 16 & 64 & 8 & 32 & \\
\hline \multicolumn{6}{|l|}{ Xerostomia } \\
\hline Mild/moderate & 12 & 48 & 17 & 68 & \multirow{2}{*}{0.03} \\
\hline \multirow[t]{2}{*}{ Severe } & 13 & 52 & 8 & 32 & \\
\hline & \multicolumn{2}{|c|}{ During RT } & \multicolumn{2}{|c|}{ After RT } & \\
\hline Group B $(n=24)$ & $\mathbf{N}$ & $\%$ & $\mathbf{N}$ & $\%$ & p-value* \\
\hline \multicolumn{6}{|l|}{ Mucositis } \\
\hline Grade $\leq 2$ & 5 & 20.8 & 15 & 62.5 & \multirow{2}{*}{0.002} \\
\hline Grade 3,4 & 19 & 79.2 & 9 & 37.5 & \\
\hline \multicolumn{6}{|l|}{ Pain } \\
\hline Mild/moderate & 10 & 41.7 & 19 & 79.2 & \multirow{2}{*}{0.003} \\
\hline Severe & 14 & 58.3 & 5 & 20.8 & \\
\hline \multicolumn{6}{|l|}{ Xerostomia } \\
\hline Mild/moderate & 17 & 70.8 & 20 & 83.3 & \multirow{2}{*}{ NS } \\
\hline Severe & 7 & 29.2 & 4 & 16.7 & \\
\hline
\end{tabular}

*based on McNemar's test

Weight changes

\section{Weight Changes}

They were available in 18 patients from each group (Table 3).

Group A: Loss of weight was observed in 17 patients and ranged between 2 to $29 \mathrm{~kg}$, with a mean loss of $9,8 \mathrm{~kg}$. One patient did not lose weight.

Group B: Loss of weight was observed in 18 patients and ranged between 2 to $15 \mathrm{~kg}$, with a mean loss of $8,6 \mathrm{~kg}$.

The weight loss did not differ significantly between the two groups.

\section{DISCUSSION}

The present observations, which were collected prospectively, in a consecutive patient cohort, "out in the real world", during the routine chemoradiotherapy schedule, outside the context of a clinical trial, showed that the addition of cetuximab in the chemoradiotherapy scheme did not increase the oral toxicity.

The incidence of severe oral mucositis, objectively observed, by Oral Medicine specialist, and severe pain and xerostomia, self-reported by the patients, in group B, who received RT, cisplatin and cetuximab, did not differ significantly, when compared to those of Group A, who received RT and cisplatin, both during and after the completion of chemoRT.

The RT unplanned breaks and the RT interruptions due to severe mucositis did not differ significantly between the two patient groups, either.

Similar numbers of patients, in both groups, received antifungals and antivirals. The use of anti-infectious medications, being similar in the two groups, further indicated, though indirectly, that cetuximab did not increase the local, mucosal toxicity, since oral mucosal infections consist a significant part of oral toxicity in cancer patients [19-21, 33$38,42]$.

The prevalence of severe oral mucositis, pain and xerostomia observed during the course of chemoRT were significantly reduced in both groups after the completion of chemoRT, attributed to the administration of antifungal and antiviral treatment and prophylaxis, as has peviously been shown $[20,21,36,39,40]$.

The patients, in the two groups, did not differ significantly in most characteristics, such as the gender, age, tumor histological diagnosis and tumor size.

The numerical difference in the median age, although not significant, represents a weakness, which is related to the non-randomized controlled study design. It is not known how or whether the younger age of the patients in Group B as opposed to Group A (57 vs. 63 years) could have affected the oral toxicity and the tolerance of the patients to the combination of cetuximab, cisplatin and RT.

The significantly higher number of patients in group B (cetuximab, cisplatin and RT) without nodal involvement as opposed to Group A (8 vs. 2 patients) represents another weakness of the non randomized study design. On the other hand, significantly more patients from Group B were irradiated with a $2.0 \mathrm{~Gy}$ daily RT dose $(20 v s .8$ patients). The limited number of patients in both groups did not, however, allow for further comments and evaluations.

Cetuximab was well tolerated in our patients, as in previous reports, where cetuximab was added to RT $[8,15$, 16] or to platinum-based chemotherapy $[9-11,13]$ or to platinum-based chemotherapy and 5-FU $[12,14,17]$ and it did not increase the common toxic effects. Significant adverse effects, as they had been previously reported when cetuximab was administered concurrently with radiotherapy and platinum-based chemotherapy $[18,22]$, were not observed in the present study. 
Interestingly, a great majority of patients in both groups ( $76 \%$ from Group A and $62.5 \%$ from Group B) were in the advantageous tumor size T1 or T2 of their cancer. Furthermore, all patients were not previously treated and had no serious co-morbidities. This is in contrast to the patients of previous reports, who had locoregionally advanced head and neck cancer or previously treated recurrent and/or metastatic cancer, with potentially compromised nutrition and well being [8-17, 18, 22]. Although, as is generally accepted [1], careful selection of patients is critical in chemoRT, the extent to which the early stage of the cancer patients in the present report might have influenced toxicity, cannot be evaluated in this setting. It would be interesting to assess, in a next step, given the increasing use of cetuximab, whether patients with different stages in their cancers exhibit different tolerance values and toxicities after cetuximab administration.

Seventy per cent of the patients of both groups, in the present study, received antifungals and antivirals during the course of chemoRT, according to the policy of our Center. The need and the use of antifungals and antivirals have not been mentioned in the previous reports [8-17, 18, 22].

Local, oral mucosal infections and their potential systemic dissemination, especially in patients, compromised with advanced cancers, previous treatments and serious comorbidities, were not clearly ruled out in previous studies.

The severe adverse effects reported by Pfister et al. [22], could be related, at least in two of the cases (bacteremia and pneumonia), to systemic infection disseminated from a possible oral site-mucosal infection, superimposed on oral mucositis, while HSV-1 infection could be the cause of severe labial toxicity reported by Pryor et al. [18]. In addition, 3 of the 13 patients reported by Pryor et al. [18] had dysphagia and severe weight loss before the commencement of treatment.

The high incidence of rash and dermatitis of our patients $(62.5 \%$ of the Group B) is in agreement with the recent report by Giro et al. [43]. The acne-like rash and dermatitis, observed in our patients was adequately managed, according to the suggested guidelines [41]. Severe skin reactions as reported in the cases by Berger et al. [44] and by Budach et al. [45] were not observed.

In conclusion, no statistical differences for the toxicities under study were observed. Cetuximab did not increase the severity of the oral mucositis, pain and xerostomia in head and neck cancer patients, who received chemoradiotherapy, with limitations of the current study design and its limited number of patients. A large-scale randomized controlled trial is needed to confirm the above results.

Risk factors, including co-morbidities and the extent of locally advanced head and neck cancer stage, potentially compromising the patient, and other factors should be studied, identified and taken into account, in order to use cetuximab at an optimal setting for the optimal patient benefit.

\section{REFERENCES} Curr Opin Oncol 2002; 14: 323-29.
[2] Jain P, Kumar P, Pai RV, Parikh MP. Neoadjuvant chemotherapy or chemoradiotherapy in head and neck cancer. Indian J Cancer 2008; 45: 83-9.

[3] Maguire PD, Meyerson MB, Neal CR, et al. Toxic cure: hyperfractionated radiotherapy with concurrent cisplatin and fluoracil for the stage III and IVa head-and-neck cancer in the community. Int J Radiat Oncol Biol Phys 2004; 58: 698-704.

[4] Mendelson J, Baselga J. Status of epidermal growth factor receptor antagonists in the biology and treatment of cancer. J Clin Oncol 2003; 21: 2787-99.

[5] Li S, Schmitz K, Jeffrey P, Wiltzius J, Kussie P, Ferguson K. Structural basis for inhibition of the epidermal growth factor receptor by cetuximab. Cancer Cell 2005; 7: 301-11.

[6] Temam S, Kawaguchi H, El-Naggar, et al. Epidermal growth factor receptor copy number alterations correlate with poor clinical outcome in patients with head and neck cancer. J Clin Oncol 2007; 25: $2164-70$.

[7] Bier H, Hoffmann T, Haas I, van Lierop A. Anti (epidermal growth factor) receptor monoclonal antibodies for the induction of antibody-dependent cell-mediated cytotoxicity against squamous cell carcinoma lines of the head and neck. Cancer Immunol Immonother 1998; 46: 167-73.

[8] Robert F, Ezekiel MP, Spencer SA, et al. Phase I study of antiepidermal growth factor receptor antibody cetuximab in combination with radiation therapy in patients with advanced head and neck cancer. J Clin Oncol 2001; 19: 3234-43.

[9] Baselga J, Trigo JM, Bourhis J, et al. Phase II multicenter study of the antiepidermal growth factor receptor monoclonal antibody cetuximab in combination with platinum-based chemotherapy in patients with platinum-refracory metastatic and/or recurrent squamous cell carcinoma of the head and neck. J Clin Oncol 2005; 23: 5568-77.

[10] Chan ATC, Hsu MM, Goh BC, et al. Multicenter, phase II study of cetuximab in combination with carboplatin in patients with recurrent or metastatic nasopharyngeal carcinoma. J Clin Oncol 2005; $23: 3568-76$

[11] Herbst RS, Arqutte M, Shin DM, et al. Phase II multicenter study of the epidermal growth factor receptor antibody cetuximab and cisplatin for recurrent and refractory squamous cell carcinoma of the head and neck. J Clin Oncol 2005; 23: 5578-87.

[12] Bourhis J, Rivera F, Mesia R, et al. Phase I/II study of cetuximab in combination with cisplatin or carboplatin and flouroracil in patients with recurrent or metastatic squamous cell carcinoma of the head and neck. J Clin Oncol 2006; 24: 2866-72.

[13] Vermoken JB, Trigo J, Hitt R, et al. Open-label, uncontrolled, mutlicenter phase II study to evaluate the efficacy and toxicity of cetuximab as a single agent in patients with recurrent and/or metastatic squamous cell carcinoma of the head and neck who failed to respond to platinum-based therapy. J Clin Oncol 2007; 25: 2171-77.

[14] Vermorken J, Mesia R, Rivera F, et al. Platinum-based chemotherapy plus cetuximab in head and neck cancer. N Engl J Med 2008; 359: 1116-27.

[15] Bonner J, Harari P, Girald J, et al. Radiotherapy plus cetuximab for squamous-cell carcinoma of the head and neck. N Engl J Med 2006; 354: 567-78.

[16] Curran D, Giralt P, Harari P, et al. Quality of life in head and neck cancer patients after treatment with high- dose radiotherapy alone or in combination with cetuximab. J Clin Oncol 2007; 25: 2191-97.

[17] Rivera F, Garcia-Castano A, Vega N, Vega-Villegas ME, Gutierrez-Sanz L. Cetuximab in metastatic or recurrent head and neck cancer: the EXTREME trial. Expert Rev Anticancer Ther 2009; 9: 1421-28.

[18] Pryor D, Porceddu S, Burmeister B, et al. Enhanced toxicity with concurrent cetuximab and radiotherapy in head and neck cancer Radiother Oncol 2009; 90: 172-76.

[19] Djuric M, Jankovic L, Jovanovic T, et al. Prevalence of oral herpes simplex virus reactivation in cancer patients: a comparison of different techniques of viral detection. J Oral Pathol Med 2009; 38: 167-73.

[20] Nicolatou-Galitis O, Dardoufas K, Markoulatos P, et al. Oral pseudomembranous candidiasis, herpes simplex virus-1 infection, and oral mucositis in head and neck cancer patients receiving radiotherapy and granulocyte-macrophage colony-stimulating factor (GM-CSF) mouthwash. J Oral Pathol Med 2001; 30: 471-80. 
[21] Nicolatou-Galitis O, Athanassiadou P, Kouloulias V, et al. Herpes simplex virus-1 (HSV-1) infection in radiation-induced oral mucositis. Support Care Cancer 2006; 14: 753-62.

[22] Pfister D, Su B, Kraus D, et al. Concurrent cetuximab, cisplatina and concomitant boost radiotherapy for locoregionally advanced, squamous cell head and neck cancer: a pilot phase II study of a new combined-modality paradigm. J Clin Oncol 2006; 24: 1072- 78.

[23] Trotti A, Bellm L, Epstein J, et al. Mucositis incidence, severity and associated outcomes in patients with head and neck cancer receiving radiotherapy with or without chemotherapy: a systematic literature review. Radiother Oncol 2003; 66: 253-62.

[24] Elting LS, Cooksley C, Chambers M, Garden A. Risk, outcomes and costs of radiation-induced oral mucositis among patients with head and neck malignancies. Int J Radiat Oncol Biol Phys 2007; 68: $1110-20$

[25] Elting L, Keefe D, Sonis S, et al. Patient-reported measurements of oral mucositis in head and neck cancer patients treated with radiotherapy with or without chemotherapy. Cancer 2008; 113: 2704-13.

[26] Rosenthal D. Consequences of mucositis-induced treatment breaks and dose reductions on head and neck cancer treatment outcomes. J Support Oncol 2007; 5: 23-31.

[27] Russo G, Haddad R, Posner M, Machtay M. Radiation treatment breaks and ulcerative mucositis in head and neck cancer. Oncologist 2008; 13: 886-98.

[28] Murphy B. Clinical and economic consequences of mucositis induced by chemotherapy and/or radiation therapy. J Support Oncol 2007; 5: 13-21.

[29] Nonzee NJ, Dandade AN, Markossian T, et al. Evaluating the supportive care costs of severe radiochemotherapy-induced mucositis and pharyngitis. Cancer 2008; 113: 1446-52.

[30] Sonis ST, Elting LT, Keefe D, et al. Perspectives on cancer therapy-induced mucosal injury. Cancer 2004; 100(9 Suppl): 199525.

[31] Sonis S. Pathobiology of oral mucositis: novel insights and opportunities. J Support Oncol 2007; 5: 3-11

[32] Lalla R, Sonis S, Peterson D. Management of oral mucositis in patients with cancer. Dent Clin North Am 2008; 52: 61-77.

[33] Epstein JB, Freilich MM, Le ND. Risk factors for orofaryngeal candidiasis in patients who receive radiation therapy for malignant conditions of the head and neck. Oral Surg Oral Med Oral Pathol 1993; 76: 169-74.
[34] Dahiya MC, Redding SW, Dahiya RS, et al. Oropharyngeal candidiasis caused by non-albicans yeast in patients receiving external beam radiotherapy for head-and-neck cancer. Int J Radiat Oncol Biol Phys 2003; 57: 79-83.

[35] Nicolatou-Galitis O, Sotiropoulou-Lontou A, Velegraki A, et al. Oral candidiasis in head and neck cancer patients receiving radiotherapy with amifostine cytoprotection. Oral Oncol 2003; 39: 397-401.

[36] Nicolatou-Galitis O, Velegraki A, Sotiropoulou-Lontou A, et al. Effect of fluconazole antifungal prophylaxis on oral mucositis in head and neck cancer patients receiving radiotherapy. Support Care Cancer 2006; 14: 44-51.

[37] Paula CR, Carmeli M, Birman EG, Siquiera AM. Oral yeasts in patients with cancer of the mouth, before and during radiotherapy. Mycopathologia 1990; 112: 119-24

[38] Correia Jham B, Franca EC, Oliveira RR, et al. Candida ora colonization and infection in Brazilian patients undergoing head and neck radiotherapy: a pilot study. Oral Surg Oral Med Oral Pathol Oral Radiol Endod 2007; 103: 355-58.

[39] Mücke R, Kaben U, Libera $\mathrm{T}$, et al. Fluconazole prophylaxis in patients with head and neck tumors undergoing radiation and radiochemotherapy. Mycoses 1998; 41: 421-23.

[40] Koc M, Aktas E. Prophylactic treatment of mycotic mucositis in radiotherapy of patients with head and neck cancer. Jpn J Clin Oncol 2003; 33: 557-60.

[41] Bernier J, Bonner J, Vermoken JB, et al. Consensus guidelines for the management of radiation dermatitis and coexisting acne-like rash in patients receiving radiotherapy plus EGFR inhibitors for the treatment of squamous cell carcinoma of the head and neck. Ann Oncol 2008; 19: 142-49.

[42] Lalla RV, Latortue MC, Hong $\mathrm{CH}$, et al. A systematic review of oral fungal infections in patients receiving cancer therapy. Support Care Cancer 2010; [Epub ahead of print].

[43] Giro C, Berger B, Bolke E, et al. High rate of severe radiation dermatitis during radiation therapy with concurrent cetuximab in head and neck cancer: Results of a survey in EORTC institutes. Radiother Oncol 2009; 90: 166-71.

[44] Berger B, Belka C. Severe skin reaction secondary to concomitant radiotherapy plus cetuximab. Radiat Oncol 2008; 3: 5 .

[45] Budach W, Bolke E, Homey B. Severe cutaneous reaction during radiation therapy with concurrent cetuximab. N Engl J Med 2007; 357: $514-15$. 